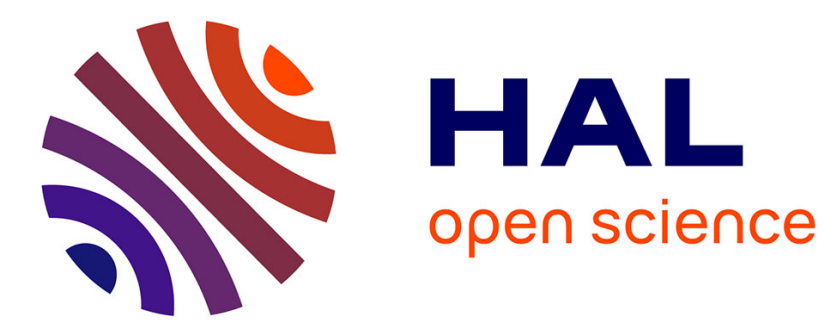

\title{
The Prince's tears, a large cable-driven parallel robot for an artistic exhibition
}

Jean-Pierre Merlet, Yves Papegay, Anne-Valérie Gasc

\section{To cite this version:}

Jean-Pierre Merlet, Yves Papegay, Anne-Valérie Gasc. The Prince's tears, a large cable-driven parallel robot for an artistic exhibition. ICRA, May 2020, Paris, France. hal-03508606

\section{HAL Id: hal-03508606 \\ https://hal.inria.fr/hal-03508606}

Submitted on 3 Jan 2022

HAL is a multi-disciplinary open access archive for the deposit and dissemination of scientific research documents, whether they are published or not. The documents may come from teaching and research institutions in France or abroad, or from public or private research centers.
L'archive ouverte pluridisciplinaire HAL, est destinée au dépôt et à la diffusion de documents scientifiques de niveau recherche, publiés ou non, émanant des établissements d'enseignement et de recherche français ou étrangers, des laboratoires publics ou privés. 


\title{
The Prince's tears, a large cable-driven parallel robot for an artistic exhibition
}

\author{
J-P. Merlet ${ }^{1}$, Y. Papegay ${ }^{1}$, Anne-Valérie Gasc ${ }^{2}$
}

\begin{abstract}
This paper presents the development and results of a large 3 d.o.f cable-driven parallel robot (CDPR) that has been extensively used between June and August 2019 for an artistic exhibition. The purpose of the exhibition was to 3D print a wall of glass powder, which will slowly collapse after the deposit of each layer. Positioning control on the assigned trajectory was an issue because of the CDPR geometry imposed by the specific configuration of the exhibition place. We describe how this problem was solved using a combination of cable length estimation based on the winch rotation measured by encoder, together with 3 on-board lidars that were used to provide a measure of the robot position. To the best of our knowledge this is the first time that such method was used for controlling a large CDPR. This CDPR has run for 174 hours since 6/18/2019, averaging a run time of $4 \mathrm{~h} 15 \mathrm{mn}$ per day. The $3 \mathrm{D}$ printing of the wall started on $7 / 18 / 2019$ and stops on 8/31/2019. During this period the robot was used for 32 days with an average of $2 \mathrm{~h} 18 \mathrm{mn}$ run-time per day. The robot has traveled on a total distance of 4757 meters, of which 3893 meters on the assigned trajectory. During the period 76 layers have been deposited, representing a mass of $\mathbf{1 . 5}$ tons of glass powder.
\end{abstract}

Index terms: cable-driven parallel robot, kinematics, art

\section{INTRODUCTION}

In March 2018 Y. Papegay, a member of the HEPHAISTOS team, met the exhibition curator Emmanuelle Chiappone-Piriou, an architect and architecture historian, during a seminar on architecture and numerical sciences, while he was working on the use of cable-driven parallel robot (CDPR) for the construction industry. CDPR moves a load by coiling/uncoiling cables whose ends are connected both to the ground and to the load. This curator was in touch with the artist Anne-Valérie Gasc, which was considering to present an exhibition called the Prince's tears: vitrifications. Anne-Valérie Gasc is an associate professor at ENSA-M (École Nationale Supérieure d'Architecture de Marseille). She is leading since 2014 a large project called Prince's tears and was invited in April 2019 at the contemporary art center of Amilly to finalize AN exhibition. Its purpose was to deposit several layers of glass powder (density: $1.6 \mathrm{~kg} / \mathrm{l}$ ) along a given $3 \mathrm{D}$ trajectory. The trajectory was defined by a curve in the $x-y$ plane that has to be followed at various altitudes. For a given altitude transition poses were defined on the $x-y$ curve, where the system should either start or stop depositing the powder. The system was designed to be installed in the main hall of the art center les Tanneries located at Amilly, $100 \mathrm{~km}$ south of Paris. This hall is a large place, whose central part is 22 meters of length, 8 meters

\footnotetext{
${ }^{1} \mathrm{HEPHAISTOS}$ project, Inria Sophia-Antipolis, France Jean-Pierre.Merlet/ Yves.Papegayeinria.fr, ${ }^{2}$ ENSA-M (École Nationale Supérieure d'Architecture de Marseille)
}

of width for a height of 5.3 meters and surrounded by 12 pillars, 6 on each side (figure 1).

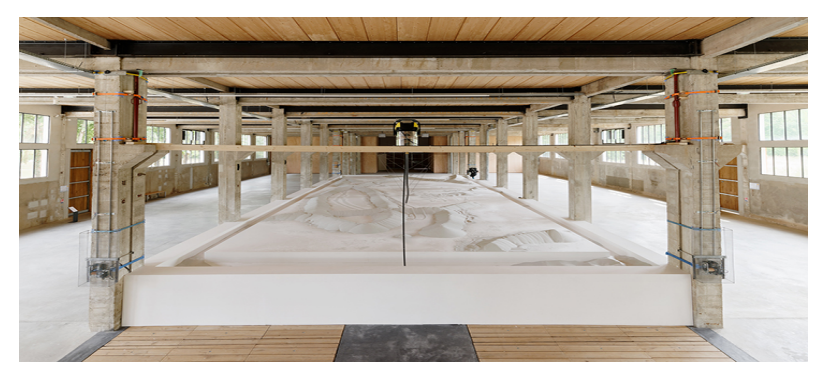

Fig. 1. The exhibition place with the $20.8 \times 7.3$ meters platform.

For the deposit of the powder the idea was to move a drum filled with about $30 \mathrm{~kg}$ of powder along the trajectory, which therefore requires only translation motion. The place on which the powder should be deposited should be a white planar platform whose height is 1 meter above the hall ground (a height that will have a negative influence as will be seen later on), while the desired $x-y$ curve, whose total length is about 50 meters, will be enclosed in a rectangle of size $20.8 \times 7.3$ meters. As soon as the robot started to perform the layers it was forbidden to walk on the platform, that was to remain immaculate. Regarding height, the difference between two successive layers was fixed to $10 \mathrm{~cm}$, the final layer being at 2 meters over the platform. The velocity of the drum was not quantified but should be such that a sufficient number of layers should be deposited during the exhibition time i.e. between June 22 and August 31. Powder storage tanks were supposed to be located on both ends of the central part, with an automatic system located on the border of the platform, whose role will be to refill the drum as soon as it was empty. Besides these technological constraints, an artistic constraint was that the system moving the drum was to present a very low visual intrusivity in order to offer an almost unobstructed view of the powder wall. Furthermore the available budget was very limited, especially regarding the development of the system.

Y. Papegay met the artist during the autumn of 2018 and it was decided that the INRIA HEPHAISTOS team will be a partner of this project that, beside being an art project, will also become a scientific one. The team has experience in deploying large CDPR such as our MARIONET-CRANE prototype [1] which, beside being a 6 d.o.f robot, was covering an even larger workspace than the one required for the exhibition. The 10 years old hardware of MARIONET- 
CRANE was supposed to be adapted for this project. Our scientific objectives was first to test moving a CDPR over a long period of time in quasi-industrial conditions under the control of non-experts and, second, to extensively instrument the robot for a better understanding of its behavior during this period, with possible future consequences on its theoretical modeling and control. The development of the system requires to:

- design the control of a CDPR whose geometry was fixed (meaning that its kinematics features were imposed). The absolute accuracy along the $x-y$ axes was not that important while the accuracy on the $z$ axis was fixed to $\pm 5 \mathrm{~cm}$ in order to respect the layer disposition and to avoid that the drum hit the platform

- design on-board systems for managing the powder flow and detecting that the drum was empty

- design an automatic system for refilling the drum

- develop a communication system between the computer that was managing the CDPR and the on-board computer of the drum.

\section{THE CDPR}

\section{A. Notations}

As seen in the introduction this exhibition was requiring a 3 d.o.f. suspended CDPR whose workspace shall include the $x-y$ curve and the various layers. The geometry of the exhibition place and the workspace constraint imposed a CDPR with 4 cables, all connected at the same point $B$ on the drum so that we have actuation redundancy. We will denote by $A_{i}$ the winch output point of the $i$-th cable and by $B$ the connecting point of the cable on the drum. The cable will be assumed to be ideal (no elasticity and no sagging) and $\rho$ will denote the cable length (i.e. the distance between $A$ and $B$ ). The exhibition place has led us to put the 4 winches at the foot of the 4 extreme pillars with a pulley system leading to the $A$ points close to the ceiling. A reference frame is defined with origin at the center of the platform, the $x$ axis being directed along the largest dimension of the platform and the $y$ axis along the smallest one. The coordinates of the $A$ points, in meters, in this reference frame are given below

\begin{tabular}{c||c|c|c|c|}
\hline & $A_{1}$ & $A_{2}$ & $A_{3}$ & $A_{3}$ \\
\hline \hline$x$ & -10.4 & -10.4 & 10.4 & 10.4 \\
\hline$y$ & 3.64 & -3.64 & -3.64 & 3.64 \\
\hline$z$ & 5.1 & 5.1 & 5.1 & 5.1 \\
\hline
\end{tabular}

The coordinates of the $B$ point in the reference frame has be chosen to parametrize the pose of the robot.

\section{B. Kinematics and statics}

It is well known that a suspended CDPR with 4 ideal cables connected at the same point will have, at a given pose, at most 3 cables under tension, the other one being slack. Furthermore at this pose there are two triplet of cables that may be under tension, the other triplets having negative tensions. Assuming ideal cables the inverse kinematics (i.e. computing the $\rho$ for a given pose) is trivial: the coordinates in the reference frame of $B, A_{i}$ are known and we have
$\rho_{i}=\left\|\mathbf{A}_{\mathbf{i}} \mathbf{B}\right\|$. The direct kinematics, DK, (i.e. determining the robot pose for a given set of $\rho$ ) is simple for a parallel robot. For a given triplet of cables under tension there are 2 solutions for the DK but one of them to a pose that is over the $A$ : as the cables cannot push this solution may be discarded. As we have usually 2 triplets of cables that may be under tension we have therefore 2 solutions for the DK. We will see later on that we are able to have a good estimate of the robot pose at a given sampling time, so that at the next one, at which new cable lengths are measured, we will retain as the current pose the DK solution that is the closest to the previous estimation.

It is also of interest to determine the relation between variations $\Delta \rho$ of the cable lengths and the corresponding variations of the platform pose $\Delta \mathbf{X}$. For a given triplet of cables we have

$$
\Delta \rho=\mathbf{J}^{-1} \Delta \mathbf{X}
$$

where $\mathbf{J}^{-1}$ is the inverse kinematic jacobian which is a $3 \times$ 3 matrix. If $x, y, z$ denote the coordinates of $B$ and $x_{i}, y_{i}, z_{i}$ represent the coordinates of $A_{i}$, then the $i$-th row of $\mathbf{J}^{-\mathbf{1}}$ is

$$
\left(\left(\frac{x-x_{i}}{\rho_{i}} \quad \frac{y-y_{i}}{\rho_{i}} \quad \frac{z-z_{i}}{\rho_{i}}\right)\right)
$$

Equation (1) allows one to determine the influence on the positioning of the robot of the errors on the cable length measurements. Regarding statics, the external wrench applied on the robot is only due to the gravity and may therefore be written as $\mathcal{F}=(0,0,-m g)^{T}$, where $m$ is the known mass of the drum. If $\tau$ denotes the cable tensions for a given triplet of cables we have

$$
\mathcal{F}=\mathbf{J}^{-\mathbf{T}_{\tau}}
$$

Equation (2) therefore allows one to calculate the cable tensions for a given pose of the robot. An approach based on interval analysis has allowed us to determine what should be the maximal tensions in the cables over the workspace, taking into account the variable mass of the drum.

We have determined that the cable lengths will lie in the range [4.38,19.76] meters for any pose in the desired workspace. Over this worskspace the maximal cable tension will be $1263 \mathrm{~N}$ for a drum mass of $50 \mathrm{~kg}$, while the mean value of the cable tensions over the whole workspace will be $590 \mathrm{~N}$. As a consequence we decided to use Dyneema cables with diameter $3 \mathrm{~mm}$ and a breaking tension of $11000 \mathrm{~N}$. We also established the influence on the positioning of the CDPR of an error of $1 \mathrm{~cm}$ on the cable length measurements. The maximal pose variation was determined to be $1.29 \mathrm{~cm}$ for $\Delta x, 5.28 \mathrm{~cm}$ for $\Delta y$ and $5.15 \mathrm{~cm}$ for $\Delta z$, while their mean values were respectively $1.08,2.59,3.61 \mathrm{~cm}$. The large amplification factor between $\Delta \rho$ and $\Delta \mathbf{X}$ for the $y, z$ axes is due to the geometry of the robot (thin along the $y$ axis compared to the $x$ dimension) and to the imposed height of the robot, which leads to cables that are close to the horizontal. The cable angle with the horizontal was established to lie in the range $[6.35,50.18]$ degrees with a mean value of 16.79 degrees. Our objective was to reach an accuracy of $\pm 10 \mathrm{~cm}$ along the $x, y$ axis and $\pm 3 \mathrm{~cm}$ along 
the $z$ axis. According to the previous figures, this implies that the cable lengths will have to be measured with an accuracy of $3 / 5.15=0.57 \mathrm{~cm}, 0.03 \%$ of the cable length range, an unrealistic value. Note also that for a given pose the CDPR characteristics differ according to the triplet of cable under tension. For example at the pose $(500,100,212)$ the cable tension are $(698,130,703) \mathrm{N}$ for the triplet $(1,3,4)$ and $(679,460,417) \mathrm{N}$ for the triplet $(2,3,4)$. Similarly the $\Delta x, \Delta z$ change according to the triplet: $(1.157,3.1268) \mathrm{cm}$ for $(1,3,4)$ and $(1.087,3.175) \mathrm{cm}$ for $(2,3,4)$.

\section{The ACTUATION SYSTEM}

As mentioned previous we have recycled the winches of our MARIONET-CRANE prototype, which were designed to coil about 300 meters of steel cable of $6 \mathrm{~mm}$ of diameter using Parvalux PBL60-118 motors with $0.32 \mathrm{Nm}$ torque. Most middle-sized CDPR uses a winch with a drum coiling mechanism that moves the cable along a spiral guide on the drum [2], [3]. If the coiling is perfect, then there is a one-toone relationship between the rotation of the drum (which is measured by an encoder) and the coiled cable length. However this approach has the drawback to impose a single cable layer on the drum. As the drum radius is limited by the maximal motor torque, the single cable layer impose strong restriction on the maximal cable length. Our winches just coil the cable on the fly, possibly on several layers (figure 2). The drawback of these winches is that we have no control on

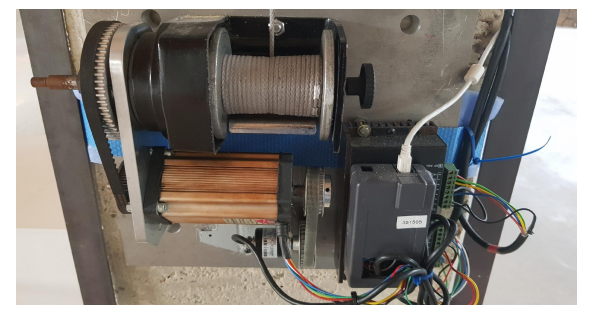

Fig. 2. The winch system

the coiling process, which changes the drum diameter and therefore the relationship between the drum rotation and the cable length. Therefore we cannot reach the required $0.56 \mathrm{~cm}$ cable length measurement accuracy with only the encoder measures, so that a new approach was necessary.

\section{IMPROVING THE CDPR POSITIONING ACCURACY}

For reaching the required accuracy a first approach is to improve the measurements of the cable lengths.

\section{A. The marks system}

For this purpose we have first applied an approach that was proposed in [4]. Small aluminium stickers (that can be seen on figure 2), called em marks, were put on the cables at specific calibrated lengths. Cable guides, called stations, were installed on the pillar, the cable going through 2 rollers covered by an aluminium foil. When a mark goes through the rollers, it establishes an electrical contact that can be detected. Provided that the mark number and the location of the rollers with respect to the winch are known, then the cable length is determined. Using 8 marks on the cable and 3 stations it is possible to show that the cable length changes between two successive detection events lies in the range $[30,50] \mathrm{cm}$. Furthermore as soon as two successive events involve different stations or marks we have both the exact cable length change and the corresponding rotation angle of the winch, as measured by the encoder, this allowing to update the current winch radius. This estimation is used to determine the cable length based on the encoder measures in-between detection events. Tests have shown that this approach was leading to an accuracy for the cable length better than $1 \mathrm{~cm}$ and a good positioning accuracy of the CDPR. Unfortunately we noticed that over time the stickers were sliding on the cables, leading to a derive of the measurements, so that the system will have required a regular maintenance that was not possible in the exhibition context.

\section{B. Using on-board sensing}

We may have considered using a vision system to determine the cable directions, a method that has be proven to be efficient for small parallel robots [5] and has been tentatively used for CDPR [6], but with less convincing result. But the system size and the varying illumination condition (including the possibility of working at night with only lights directed toward the deposit platform) prohibited this approach. Another possibility, often mentioned in the CDPR community, is to add sensing information for example measuring the cable direction at $B$ or using IMUs on the platform to get its orientation [7], [8]. Although the quality of these measurements may be very good [9], they have never been used in practice for large CDPR, to the best of our knowledge. For this project we choose a simpler approach by adding three RPLIDAR A1M8 lidars on the drum (figure 3), having a measuring range between $0.15 \mathrm{~m}$ and $10 \mathrm{~m}$, an accuracy of about $5 \mathrm{~mm}$ and able to perform a 360 degree scan with an angular resolution of 0.5 degree in less than one second. A vertical lidar is used to determine the height of

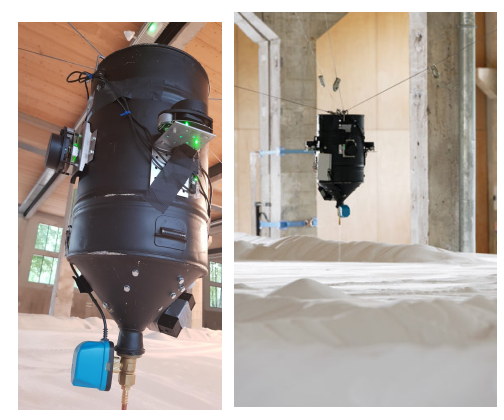

Fig. 3. The drum with its three lidars, the vanne (in blue) and the fit-pc. On the right a powder deposit

the drum, acquiring measures in a plane that is perpendicular to the $x$ axis with a scan angle in the range $[-10,10]$ degrees around the vertical. Let $P$ be the point among the measures leading to the lowest $z, z_{\min }$ and the set $S$ of measures with a $z$ value in the range $\left[z_{\text {min }}, z_{\min }+1 \mathrm{~cm}\right]$. We assume that the 
points of $S$ were all located on the deposit platform and a least square method was used to determine the $z$ coordinate of the drum. The two other lidars, located respectively on the

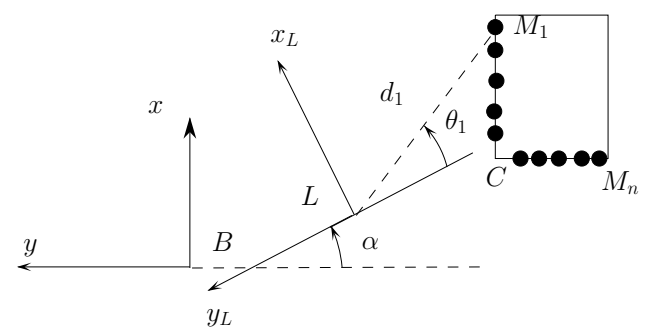

Fig. 4. Notation for the lidar measurements

left and right side of the drum, were designed to perform an horizontal scan. Being given the lidar range it was not expected that points on all the pillars will be measured but as the distance between the pillars is about 3 meters, we may assume that points on 2 pillars may be measured, either on the left or right side or on both sides. Consider a lidar located at $L$ with known coordinates $\left(x_{l}, y_{l}\right)$ in the frame $\mathcal{R}_{B}=(B, \mathbf{x}, \mathbf{y})$. A local frame $\left(L \mathbf{x}_{\mathbf{L}}, \mathbf{y}_{\mathbf{L}}\right)$ is attached to the lidar and the angle between $\mathbf{x}$ and $\mathbf{x}_{\mathbf{L}}$ is denoted by $\alpha$, whose value is small but unknown (figure 4).

The lidar measurements are a set of pairs $\left(d_{i}, \theta_{i}\right)$, where $d_{i}$ is the distance and $\theta_{i}$ the angular measure. Such a pair corresponds to a point $M_{i}$ on the pillar, some of them having a constant $x=x_{s}$ coordinate in the $\mathcal{R}_{B}$ frame, while the other have a constant $y=y_{s}$ coordinate. In the lidar frame the coordinates of $M_{i}$ are $\left(d_{i} \sin \theta_{i}=x_{l}^{i}, d_{i} \cos \theta_{i}=y_{l}^{i}\right)$. The coordinates of $M_{i}$ in the $\mathcal{R}_{B}$ frame are $\left(x_{i}=\left(x_{l}^{i}+\right.\right.$ $\left.x_{l}\right) \cos \alpha-\left(y_{l}^{i}+y_{l}\right) \sin \alpha, y_{i}=\left(x_{l}^{i}+x_{l}\right) \sin \alpha+\left(y_{l}^{i}+\right.$ $\left.y_{l}\right) \cos \alpha$ ). Let $\mathcal{T}=\left\{M_{1}, \ldots, M_{n}\right\}$ be the set of $n$ measured points on a given pillar. Consider the cost function

$$
H_{k}=\sum_{j=1}^{2 \leq k \leq n}\left(x_{i}-x_{s}\right)^{2}+\sum_{j=k+1 \leq n}^{j=n}\left(y_{i}-y_{s}\right)^{2}
$$

For a given $k$ in the range $[2, n]$ this cost function characterize the difference between the $x$ coordinates of the $[1, k]$ measured points and a constant $x_{s}$ and the difference between the $y$ coordinate of the $[k+1, n]$ measures with a constant $y_{s}$. For a given $k$ the unknowns of the cost function are $\alpha, x_{s}, y_{s}$ and it is easy to show that the unknown values that minimize the cost may be determined in closed-form. As their is a limited number of points that are measured on a given pillar (between 10 and 30 according to the pillar proximity), we just consider all cost function $H_{k}$ with $k$ in $[2, n]$ and as values for $\alpha, x_{s}, y_{s}$ the one leading to the lowest residual among all $H_{k}$. The coordinate of the corner $C$ of the pillar in the $\mathcal{R}_{B}$ frame is then $\left(x_{s}, y_{s}\right)$ and by looking at the sign of $x_{s}$ we may determine if $C$ is a lower left or upper left pillar corner. The coordinate in the reference frame of these corners for all the pillars are known but at this point we don't know on which pillar lie the $M_{i}$ points. This issue can be solved by an initial calibration: when starting the system the lidars measure the corner position and deduce 6 possible poses of the drum by assuming that the corner lie on one of the 6 pillars. The end-user then select the right pose by visual inspection so that the current pose is determined. For the further measurements we still compute all 6 possibilities and retain the one which is the closest to the current pose estimate. In our system the lidars take a measurement every 4 seconds but it may happen that several successive measurements do not allow for an update of the $x, y$ coordinates of the CDPR pose, especially when the lidars are close to be in front of a pillar (but the $z$ coordinate can always be updated). In between lidar update we use the encoder measurements to estimate the cable lengths and then use the DK to compute the current pose. Assuming a perfect coiling of the winches we have established a relationship between the cable length and the winch drum radius as a set of pairs of interval values for the cable length and a corresponding winch radius. Using these data we are able to estimate what is the cable length change for a given rotation of the encoder. We then use the DK to estimate the current pose of the CDPR. As soon as a lidar measure is available we update the pose $z$ coordinate and its $x, y$ coordinates if the measure is valid. Using the inverse kinematics we update the cable lengths and therefore the winch radii.

\section{TRAJECTORY AND CONTROL}

The trajectory is defined as a set of third order polynomials depending on the time $t$ in the range $[0,1]$ for the $x, y$ coordinates. To each member of this set is associated a state for the powder deposit, on or off. We use speed control for moving along the trajectory. Being given the current CDPR pose $\mathbf{X}$, we compute a new pose $\mathbf{X}_{\mathbf{n}}$ that lie on the trajectory and is $1 \mathrm{~cm}$ away from $\mathbf{X}$. Being given the required horizontal velocity $v$ of the CDPR $(3.5 \mathrm{~cm} / \mathrm{s})$ we define the horizontal velocity $\mathbf{V}_{\mathbf{x y}}$ of the CDPR as $v \mathbf{X}_{\mathbf{n}} \mathbf{X}$. Regarding height control we use a PID controller to calculate a vertical velocity $V_{z}$ of the CDPR based on the $z$ error. Hence the desired velocity of the drum is defined as $\mathcal{V}=\left(\mathbf{V}_{\mathbf{x y}}, V_{z}\right)$. The inverse kinematic jacobian is then used to calculate the cable velocity $\dot{\rho}$ as $\mathbf{J}^{-\mathbf{1}}(\mathbf{X}) \mathcal{V}$. Then using the current estimates of the winches radii $\dot{\rho}$ is converted into a velocity vector $\omega$ for the motors, which are controlled by a PID.

\section{THE EXHIBITION}

\section{A. The initial tests and consequences}

We started installing the system on site (located $900 \mathrm{~km}$ away from our laboratory) on June 18, 2019, with a drum having a diameter of $32 \mathrm{~cm}$ and a total height of $82 \mathrm{~cm}$. At the bottom of the drum was installed a servo-vanne designed for air/water for controlling the powder flow. A light sensor was installed at the bottom of the drum to detect if the drum was empty. We have on-board a low consumption fit-pc computer (https://www.fit-pc.com/web/) that uses Phidgets to control the servo-vanne and get the light measurement. A wifi router was set up and a communication system based on a message passing scheme allows to exchange messages between the fit-pc and the master computer that is controlling the CDPR. 
We tested also the refill station system which is based on a vacuum cleaner located 3 meters over the platform with as input a pipe running into the powder storage tank and as output another pipe that leads the powder to the drum. The powder mass induces significant changes in the pose of the end of this pipe so that a good positioning of the CDPR is required for a successful refill. The total cost of the system (computers, hardware, sensors, motors and controllers without the refill apparatus) was about 3000 euros. We have not used any industrial controller system because our control laws and sensory needs were very specific but the results shows that a less expensive system may work as reliably as industrial grade hardware [10].

The system using the marks approach described in section IV-A was pre-installed. As mentioned in this section we figured out that the marks approach was not reliable after a few days of trial. Consequently we decided to install the lidar system of section IV-B in order to improve the positioning accuracy of the CDPR. Furthermore the tests have shown that the powder wall height was not going to exceed $30 \mathrm{~cm}$ as the powder was quickly sliding along the wall edge (figure 5). Consequently it was decided to move the drum at a fixed altitude, $40 \mathrm{~cm}$ over the platform, for all deposit. As the vertical lidar scans only in a range of [10,10] degree around the vertical when the CDPR is coming close to a large section of the wall the measured altitude was higher than the platform altitude, so that the CDPR $z$ motion were reflecting the wall shape, climbing when coming close to the wall and going down later on, which was perfect for avoiding hitting the layers. We also noticed some problem in the uncoiling process when the cable tension imposed by the drum was close to 0 . In that case the only tension imposed on the winch is the weight of the cable (linear density: 5 grams per meter) and the friction in the pulley system was much higher than this weight. Consequently the winch moves its drum for uncoiling the cable but the cable is not moving, which creates a cable loop that is reversing the cable coiling: although the winch was trying to uncoil the cable, its real action was to coil it. In order to avoid this problem we put counterweight on the cable about 2 meters away from the $B$ point in order to ensure a minimal tension in the cable. To save time we also decided not to use the refill station but to manually fill the drum with a powder quantity sufficient to deposit a layer on the whole trajectory and then moves to a rest pose close to the workspace border for a refill. From the rest pose the CDPR then moves along an approach trajectory with the vanne being closed until it reaches the trajectory starting point. Including the approach motion the time required to deposit a single layer was about 40 minutes. Although we rushed to make all these changes the system was not fully operational for the official opening of the exhibition on $6 / 22$ and started to perform regular deposits on July 18 with 2 to 3 deposits per day, 5 days a week.

\section{B. Results}

Between June 18 and August 31 the system worked during $174 \mathrm{~h} 18 \mathrm{mn}$ with an average of $4 \mathrm{~h} 15 \mathrm{mn}$ per day and no system maintenance was performed during this period. Between July 18 and August 31 the system worked during 32 days, $73 \mathrm{~h} 48 \mathrm{mn}$ with 45 hours on the trajectory. During this time period the total distance covered by the CDPR under cartesian motion control was 4757 meters, among which 3893 on the trajectory. The system has printed 76 layers on the trajectory, amounting to 1.5 tons of glass powder (figure 5). We have had as breakdowns an encoder axis, the servo-vanne that has to be changed twice because the powder was grinding the opening mechanism and a cable break with no consequence as the remaining cables were able to sustain the drum. The cable break was due to an error: the aluminium stickers on this cable were not removed and have accumulated at the higher pulley, creating an abrasive ball that was wearing the cable. Finally a major breakdown occurred the day before the closing of the exhibition during which the system was supposed to perform in front of various officials: one of the winch drum axis get out axially of its lodging because the ring that was blocking the axial motion break down. But the local personnel was able to weld another blocking part so that the system was able to perform flawlessly during the closing ceremony.

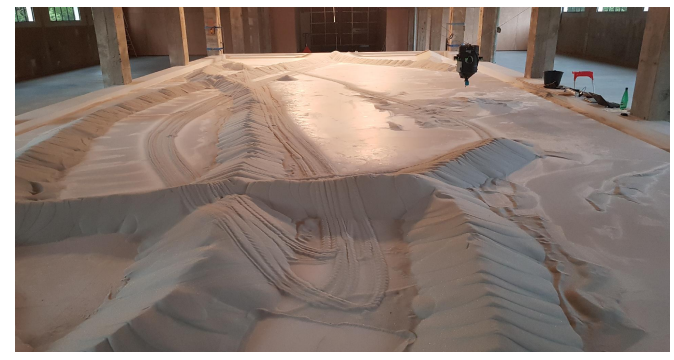

Fig. 5. The powder deposit on August 18

The poor geometrical setting allows to emphasize the importance of height for CDPR. For example if the deposit platform has been located on the ground (one meter above its current location) we will have has a maximal tension of $884 \mathrm{~N}$ in the cable (instead of $1263 \mathrm{~N}$ ), thereby allowing to reach a velocity of $5 \mathrm{~cm} / \mathrm{s}$ instead of $3.5 \mathrm{~cm} / \mathrm{s}$. The amplification factor $\Delta z / \Delta \rho$ will have been 3.6 instead of 5.15 with a mean value of 2.69 instead of 3.61 , thereby allowing to obtain a better positioning accuracy. The breakdowns may have been avoided by a minimal maintenance except for the winch axis. But the used hardware was more than 10 years old and such an accident may be expected for winches whose price is 100 euros. Nevertheless this laboratory prototype was able to perform satisfactory in quasi-industrial conditions and this work shows that CDPR are able to perform complex tasks even in adverse conditions.

Regarding on-board lidar use for CDPR control, the system has shown that good results may be obtained even with low cost lidars (100 euros apiece). The context was not the best for determining the $x, y$ coordinates of the platform with few recognizable landmarks. We have indeed noticed that up to 32 s (i.e. 8 successive lidar measurements) may be required to obtain a good estimate of these coordinates, 
thereby leading to an absolute positioning accuracy of \pm $10 \mathrm{~cm}$, which was sufficient for the task. Much better result were obtained for the $z$ coordinate with a good estimate every 4 seconds, leading to an absolute positioning accuracy of $\pm 2 \mathrm{~cm}$. A major issue was managing cable slackness. As seen previously the cable lengths are estimated according to the lidar measurements or derived from the drum rotation measures, using an estimation of the winch drum radius. For the cables under tension this estimation is accurate but much less so for slack cables. Hence our approach of the direct kinematics favor a given cable triplet so that the slack cable remains slack until the CDPR reaches a transition phase where it becomes under tension, that will be detected during the next valid lidar measurement. But we don't choose which triplet is used, although as seen in section II-B, the two possible triplets may exhibit different performances. Better performance may be obtained if the best triplet, according to some criterion, will be enforced by control (e.g. by uncoiling one cable so that we will be sure that it is slack). Managing cable slackness requires to identify which cable is becoming slack, for example by measuring the cable angles at the $B$ point as we have already shown that the use of an optical sensor for that purpose is efficient [9]. Then a bias may be used to control the length of the cable that should be slack.

The behavior of the CDPR during the whole experiment has been recorded in compressed log files for a total of $671 \mathrm{Mb}$, that are currently being processed to detect abnormalities in the CDPR behavior and for improving the control scheme. These files describe both the internal state parameters of the CDPR and, whenever available, the estimated pose of the drum obtained by the lidar system. Cable tensions have not been measured as their measurements is not reliable. The sway of the drum was not measured but visually it appears to be negligible because of the low velocity of the CDPR.

We have also make use of the vertical lidar data to obtain cross-sections of the wall in the $y-z$ plane at various $x$ values when performing a trajectory. We start with an empty set of $x$ coordinate and whenever the minimal distance between the $x$ coordinate of the drum and the set member was at least $10 \mathrm{~cm}$ we stored the lidar scan data and put the $x$ coordinate in the set. Typically we get around 105 scans for a trajectory. Figure 6 shows a typical scan obtained when the drum pose was $(697,-4) \mathrm{cm}$. We intend to use these daily scans to show the evolution of the wall during the exhibition.

\section{CONCLUSION}

We have described the use of a large CDPR prototype working in quasi-industrial conditions, averaging an use time of more than 4 hours per working days without any maintenance. The imposed design has induced severe constraints on the performances, but they had been overcomed by the first time use of on-board lidars to successfully determine the CDPR pose. This experiment confirms that CDPR may already be used for industrial task (e.g. brick building [11]), although CDPR theory is not yet complete. From a scientific viewpoint this experiment has shown the importance of

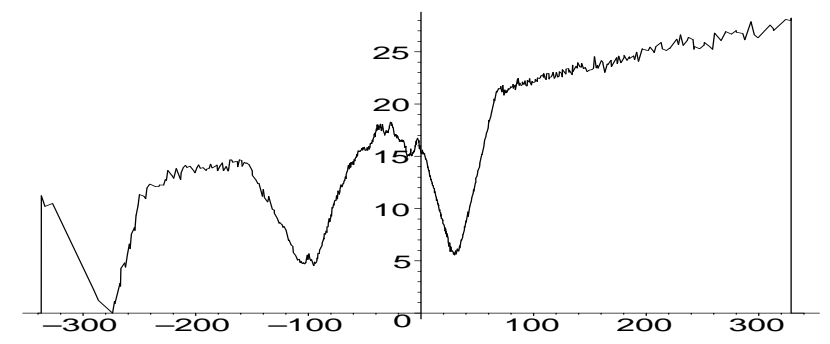

Fig. 6. $y-z$ plane cross-section of the wall for the pose $(697,-4) \mathrm{cm}$ (unit: $\mathrm{cm})$

the geometrical design for CDPR and the importance of managing cable slackness, that has to be taken into account in the CDPR kinematics. This experiment will go on as a new version of this CDPR will be exhibited during ICRA 2020 and will include additional sensors.

\section{ACKNOWLEDGMENTS}

Beside INRIA scientific and financial support, the setup of this exhibition was supported by the Les Tanneries art center, EREA Simone Weil whose students built the platform, ENSA-M (École Nationale Supérieure d'Architecture de Marseille), CNAP (Centre national des arts plastiques) and DICREAM of CNC. Special thanks to Nicolas Lemaître which manages the system during July-August 2019 and to the personnel of Amilly art center. The photos illustrating this paper are copyrighted Anne-Valérie Gasc, "Vitrifications", Photograph: Aurélien Mole.

\section{REFERENCES}

[1] J.-P. Merlet, "MARIONET, a family of modular wire-driven parallel robots," in ARK, Piran, June 28- July 1, 2010, pp. 53-62. [Online]. Available:

[2] T. Bruckmann et al., "Development of a storage retrieval machine for high racks using a wire robot," in ASME DETC, Chicago, 2012.

[3] A. Pott, Cable-Driven Parallel Robots. Theory and Application. Springer, 2018.

[4] J.-P. Merlet, "Improving cable length measurements for large CDPR using the Vernier principle," in 4th Int. Conf. on cable-driven parallel robots (CableCon), Cracow, June 30- July 4, 2019. [Online]. Available:

[5] N. Andreff, T. Dallej, and P. Martinet, "Image-based visual servoing of a Gough-Stewart parallel manipulator using leg observations," Int. J. of Robotics Research, vol. 26, no. 7, pp. 677-688, July 2007.

[6] T. Dallej et al., "Towards vision-based control of cable-driven parallel robots," in IEEE Int. Conf. on Intelligent Robots and Systems (IROS), San-Francisco, September, 25-30, 2011.

[7] X. Weber, L. Cuvillon, and J. Gangloff, "Active vibration canceling of a cable-driven parallel robot using reaction wheels," in IEEE Int. Conf. on Intelligent Robots and Systems (IROS), 2014, pp. 1724-1729.

[8] H. Yuan, E. Courteille, and D. Deblaise, "Static and dynamic stiffness analyses of cable-driven parallel robots with non-negligible cable mass and elasticity," Mechanism and Machine Theory, vol. 85, pp. 64-81, March 2015.

[9] J.-P. Merlet, "An experimental investigation of extra measurements for solving the direct kinematics of cable-driven parallel robots," in IEEE Int. Conf. on Robotics and Automation, Brisbane, May, 21-25, 2018.

[10] P. Tempel, F. Schnelle, A. Pott, and P. Eberhard, "Design and programming for cable-driven parallel robots in the german pavilion at the expo 2015," Machines, vol. 3, no. 3, pp. 223-241, 2015.

[11] Y. Wu et al., "CU-brick cable-driven robot for automated construction of complex brick structures: From simulation to hardware realisation," in 2018 IEEE International Conference on Simulation, Modeling, and Programming for Autonomous Robots (SIMPAR), 2018, pp. 166-173. 